\title{
Role of Magnetic Resonance Imaging in Localization of Acute Brachial Plexus Injury
}

\author{
Soo-Ha Kwon, MD',3; Kee-Min Yeow, MD,3; David Chwei-Chin Chuang, MD ${ }^{1,3}$; \\ Tommy Nai-Jen Chang, MD ${ }^{1,3^{*}}$
}

'Department of Plastic and Reconstructive Surgery, Chang Gung Memorial Hospital, Linkou Medical Center,

Taoyuan, Taiwan

2Department of Diagnostic Radiology, Chang Gung Memorial Hospital, Taipei Branch, Taipei, Taiwan

${ }^{3}$ Chang Gung Medical College and Chang Gung University, Taoyuan, Taiwan
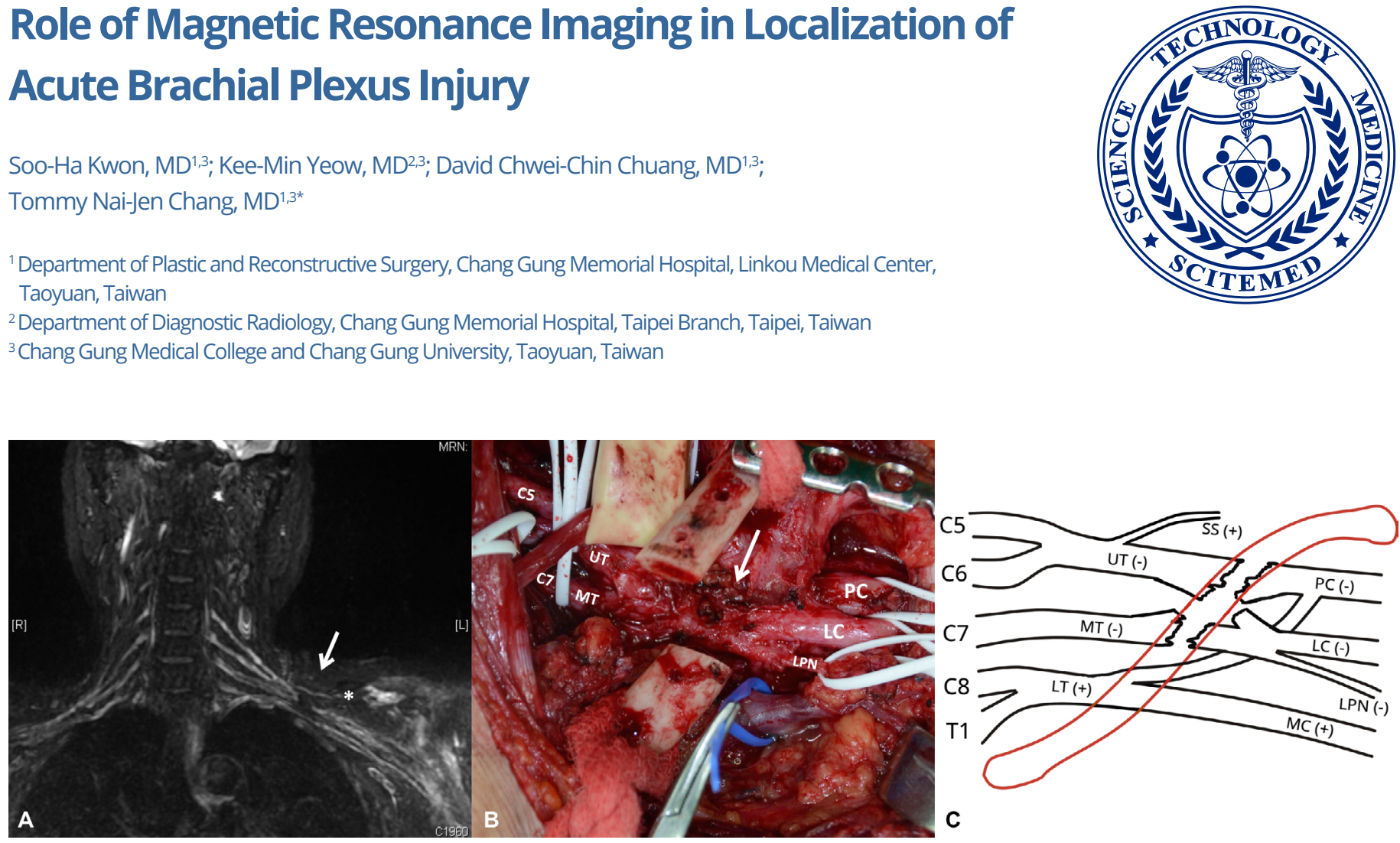

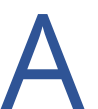

62-year-old woman presented with left upper limb weakness following involvement in a motorcycle accident. It was suspected that she had sustained acute brachial plexus injury (BPI). Neurologic (motor and sensory) examinations, motor nerve conduction velocity, electromyography results, and myelogram indicated the involvement of the upper plexus, and the possibility of avulsion injury was ruled out. However, accurate localization could not be achieved. Short tau inversion recovery (STIR) sequence magnetic resonance imaging (MRI) performed by the senior author (Yeow $\mathrm{KM}$ ) successfully targeted the lesion. A radiological examination revealed a lesion of the left upper and middle trunks at the retro-clavicular level (Chuang's level III) without clavicle fractures or injury of suprascapular nerve and lower trunk (Panel A). It was a very rare clinical scenario, which could happen in less than $5 \%$ of our series of total 1500 cases [1]. Clavicle osteotomy revealed that the lesion was compatible with the radiological finding of the intact lower trunk (Panels B and C), as confirmed by a nerve stimulator. Interpositional nerve grafts were used, and the patient regained strength gradually.

Brachial plexus imaging was traditionally challenging due to its anatomical complexity, especially, after the injury [2]. In our center, multiple sequences of MRI are performed pre-operatively for an accurate radiological diagnosis. STIR protocol provides a clear image to target the lesion precisely $[3,4]$. With the advance of the radiological setup and the experience in image analysis, nowadays, the radiological diagnosis plays a very important role in the diagnosis of acute BPI, which can shorten the operative time and make the best use of the functional nerve stump. *indicates suprascapular nerve; arrow indicates lesion site; $(+)$ indicates positive response with the nerve stimulator; (-) indicates negative response with the nerve stimulator; LC, lateral cord; LPN, lateral pectoral nerve; LT, lower trunk; MC, medial cord; MT, middle trunk; PC, posterior cord; SS, suprascapular nerve; UT, upper trunk.

\section{ARTICLE INFORMATION}

*Correspondence: Tommy Nai-Jen Chang, MD, Division of Reconstructive Microsurgery, Department of Plastic and Reconstructive Microsurgery, Chang Gung Memorial Hospital, No. 5, Fu-Hsing St. Kwei-Shan, Taoyuan, Taiwan. Email: tommynjchang@yahoo.com.tw

Received: Mar. 24, 2019; Accepted: Mar. 26, 2019; Published: Apr. 24, 2019

DOI: $10.24983 /$ scitemed.imj.2019.00111

Disclosure: This article was presented at the Second Instructional Course for Adult Brachial Plexus Injury, Linkou, Taiwan, 2017.

Conflict of Interest: The authors report no financial or other conflict of interest relevant to this article, which is the intellectual property of the authors.

Copyright (c) 2019 The Authors. This is an open-access article distributed under the terms of the Creative Commons Attribution 4.0 International License (CC-BY).

\section{REFERENCES}

1. ChuangDC.Adultbrachial plexusreconstructionwiththelevelofinjury:Reviewand personal experience. Plast Reconstr Surg 2009;124(6 Suppl):e359-369.

2. Torres C, Mailley K, Del Carpio O'Donovan R. MRI of the brachial plexus: Modified imaging technique leading to a better characterization of its anatomy and pathology. Neuroradiol J 2013;26(6):699-719.

3. Chen WC, TsaiYH, Weng HH, et al. Value of enhancement technique in 3D-T2-STIR images of the brachial plexus. J Comput Assist Tomogr 2014;38(3):335-339.

4. Tomura N, Saginoya T, Kokubun M, Tsuchiya M, Yamamoto T. T2-weighted IDEAL fast spin echo imaging of the brachial plexus: Comparison with STIR. Acta Radiol 2015;56(10):1242-1247. 